\title{
EQUIPAMENTOS DE PROTEÇÃO INDIVIDUAL UTILIZADOS POR PROFISSIONAIS DE ENFERMAGEM EM CENTROS DE MATERIAL E ESTERILIZAÇÃO
}

\author{
Personal protective equipment used by nursing \\ professionals in materials and sterilization centers \\ Equipos de protección individual utilizados por profesionales \\ de enfermería en centros de material y esterilización
}
Iolanda Beserra da Costa Santos', Maria de Fátima Gomes Santiago Cordeiroº ${ }^{2}$ Andrea Cristina de Melo ${ }^{3}$, Valdinez da Silva Lima ${ }^{4}$, Bárbara Jeane Pinto Chaves ${ }^{5}$, Paulo Emanuel Silva ${ }^{6}$

RESUMO: Objetivo: Verificar o uso de equipamento de proteção individual (EPI) por profissionais de Enfermagem em Centros de Material e Esterilização (CME). Método: Estudo quantitativo, realizado com 50 profissionais em dois hospitais de João Pessoa, Paraíba, por meio da aplicação de um questionário semiestruturado. Este trabalho foi aprovado pelo Comitê de Ética e Pesquisa (CEP), sob CAEE número 47355315.3.0000.5183. Resultados: Constatou-se que, entre os participantes, $96 \%$ são do sexo feminino, 36\% têm técnico de Enfermagem e 58\% contam mais de 10 anos de experiência. Quanto ao uso de EPI, $74 \%$ os utilizam frequentemente, $40 \%$ consideram ter pouca qualidade, $50 \%$ acham desnecessário e $68 \%$ não se adaptam às atividades. Estiveram expostos a risco ocupacional $74 \%$ dos profissionais. Participaram de treinamento sobre o tema $66 \%$ dos entrevistados e $98 \%$ reconheceram a importância da capacitação. Conclusão: Resultados apontam que os profissionais não usam regularmente os EPI, expondo-se aos riscos. Ressalta-se a necessidade de realização de uma educação permanente a fim de sensibilizá-los quanto aos cuidados.

Palavras-chave: Equipamento de proteção individual. Riscos ocupacionais. Educação em saúde.

ABSTRACT: Objective: To verify the use of personal protective equipment (PPE) by Nursing professionals in Central Sterile Supply Department (CSSD). Method: Quantitative study conducted with 50 professionals in two hospitals of João Pessoa, Paraíba, with the application of a semi-structured questionnaire. This study was approved by the Research Ethics Committee, CAEE number 47355315.3.0000.5183. Results: Among the participants, 96\% are female, $36 \%$ have technical nursing courses, and 58\% report having more than 10 years of experience. As to the use of PPE, $74 \%$ use it frequently, $40 \%$ consider it to have low quality, $50 \%$ think it is unnecessary, and $68 \%$ do not adjust to the activities. Seventy-four percent of the professionals were exposed to occupational risk. The training reached $66 \%$ of the interviewees, and $98 \%$ recognized its importance. Conclusion: The results indicate that professionals do not use PPE regularly, and are therefore exposed to risk. It is important to emphasize the need to provide continuous education in order to increase the awareness regarding care.

Keywords: Personal protective equipment. Occupational risks. Health education.

\footnotetext{
'Professora Doutora em Ciências da Saúde pela Universidade Federal da Paraíba (UFPB). Professora de Enfermagem em Centro Cirúrgico do Departamento de Enfermagem Clínica da UFPB - João Pessoa (PB), Brasil. E-mail: iolandabsc@hotmail.com

${ }^{2}$ Bióloga pela Universidade do Vale do Acaraú (UVA). Especialista em Saúde Pública pela UVA. Mestrado em Saúde Coletiva e Gestão Hospitalar pela FacNorte; Auxiliar de Enfermagem no Centro de Material e Esterilização (CME) do Hospital Universitário Lauro Wanderley (HULW) da UFPB - João Pessoa (PB), Brasil.

${ }^{3}$ Enfermeira pela Faculdade de Enfermagem e Medicina Nova Esperança (FACENE). Especialista em Saúde da Família pela Faculdades Integradas de Patos (FIP); Auxiliar de Enfermagem no CME do HULW João Pessoa (PB), Brasil.

${ }^{4}$ Enfermeira pela Faculdade Santa Emília de Rodat. Especialista em Bloco Cirúrgico, Clínica Cirúrgica e CME; Auxiliar de Enfermagem no CME do HULW - João Pessoa (PB), Brasil.

${ }^{5}$ Enfermeira pela UFPB. Especialista em Enfermagem do Trabalho; Enfermeira no CME do HULW - João Pessoa (PB), Brasil.

${ }^{6}$ Enfermeiro pela Universidade Estadual da Paraiba (UEPB). Mestre em Ciências das Religiões pela UFPB; Auxiliar de Enfermagem no CME do HULW - João Pessoa (PB), Brasil.

Rua lolanda Eloy de Medeiros, 101, bloco A, apto 1.101 - Água Fria - CEP: 58053-028 - João Pessoa (PB), Brasil.

Recebido: 29 out. 2016 - Aprovado: 15 fev. 2017

DOl: 10.5327/Z1414-4425201700010007
} 
RESUMEN: Objetivo: Verificar el uso de equipo de protección individual (EPI) por profesionales de Enfermería en Centros de Material y Esterilización (CME). Método: Estudio cuantitativo, realizado con 50 profesionales en dos hospitales de João Pessoa, Paraíba, por medio de la aplicación de un cuestionario semi-estructurado. Este trabajo fue aprobado por el Comité de Ética e Investigación (CEP), bajo CAEE número 47355315.3.0000.5183. Resultados: Se constató que, entre los participantes, el 96\% es del sexo femenino, un 36\% tiene técnico de Enfermería y un 58\% cuenta más de 10 años de experiencia. Cuanto al uso de EPI, el 74\% los utiliza frecuentemente, un $40 \%$ considera tener poca calidad, un $50 \%$ lo cree innecesario y un $68 \%$ no se adapta a las actividades. Estuvieron expuestos a riesgo ocupacional el $74 \%$ de los profesionales. Participaron de capacitación sobre el tema el 66\% delos entrevistados y el 98\% reconoció la importancia de la capacitación. Conclusión: Resultados apuntan que los profesionales no usan regularmente los EPI, exponiéndose a los riesgos. Se destaca la necesidad de realización de una educación permanente a fin de sensibilizarlos cuanto a los cuidados.

Palabras clave: Equipo de protección personal. Riesgo laborales. Educación en salud.

\section{INTRODUÇÃo}

As suposições históricas deduzem que os acidentes de trabalho tenham como ponto de partida a necessidade do homem em lutar por sua sobrevivência. Na Enfermagem, destacam-se como predisponentes para esses agravos o número insuficiente de trabalhadores, a sobrecarga de trabalho, as jornadas fatigantes, a continuidade da assistência em turnos e plantões noturnos, o desgaste físico e emocional e a capacitação técnica deficiente ${ }^{1}$. Nesse sentido, na busca para prestar uma assistência de qualidade ao ser humano, os profissionais de Enfermagem se encontram expostos a uma série de riscos - físicos, químicos, ergonômicos, psicossociais, biológicos, entre outros - , que podem causar acidentes no decorrer do turno de plantão e doenças ocupacionais ${ }^{2,3}$. A exposição ocupacional a material biológico representa um risco maior em decorrência da possibilidade de transmissão de patógenos, como o vírus da hepatite B (HBV), da hepatite C e da Imunodeficiência Adquirida (HIV), resultantes de lesões percutâneas e/ou de contato com sangue contaminado em membrana, mucosa ou pele não íntegra ${ }^{3-5}$.

Nesse contexto, a Resolução da Diretoria Colegiada da Anvisa (RDC) 15 define o Centro de Material e Esterilização (CME) "[c]omo um conjunto de elementos destinados à recepção, expurgo, preparo, esterilização, guarda e distribuição do material para unidades consumidoras dos estabelecimentos de saúde" ${ }^{\text {. }}$. Logo, esses ambientes envolvem em suas atividades diversos tipos de riscos, em especial o biológico, considerando que os trabalhadores, ao manusearem artigos contaminados, estão diretamente expostos a secreções orgânicas, o que alerta para a adoção constante de medidas de biossegurança.

Entre as precauções padrão, o equipamento de proteção individual (EPI) é usado na prevenção de acidentes de trabalho, sendo seu uso necessário em locais caracterizados como perigosos ou insalubres e naqueles que requerem higiene e segurança para sua elaboração. A resistência do profissional de Enfermagem em utilizá-lo e o seu uso incorreto são as principais barreiras para evitar risco de exposição a material biológico ${ }^{7}$.

A baixa adesão ao uso do EPI e o seu manuseio incorreto são decorrentes de fatores como desconforto, incômodo, descuido, esquecimento, falta de hábito, inadequação dos equipamentos, quantidade insuficiente, descrença de proteção quanto ao seu uso, sobrecarga de trabalho e cansaço físico. Essas condições são agravadas pela precária infraestrutura dos estabelecimentos de saúde e outros aspectos relacionados à organização do próprio trabalho, como a falta de atualização e a não existência de educação permanente em centro de material ${ }^{8}$. A adesão ao uso de EPI está intimamente relacionada à percepção dos profissionais acerca dos riscos a que estão expostos e da susceptibilidade aos mesmos. Em decorrência da contextualização descrita, questiona-se: os profissionais de Enfermagem estão usando o EPI em CME?

\section{OBJETIVO}

Este estudo teve como objetivo verificar o uso de EPI por profissionais de Enfermagem em CME. 


\section{MÉTODO}

Trata-se de um estudo exploratório com abordagem quantitativa, realizado em CME de dois hospitais públicos, localizados em João Pessoa, Estado da Paraíba, no período de janeiro a março de 2016. Os dois setores possuem características físicas e atividades semelhantes, porém se diferenciam por ser um deles de instância federal e o outro estadual.

O universo da pesquisa constitui-se por profissionais de Enfermagem - enfermeiros, auxiliares e técnicos em Enfermagem - do CME dos hospitais mencionados, dimensionada em $100 \%$, totalizando 50 entrevistados, sendo 30 do Hospital Universitário Lauro Wanderley (HULW) e 20 do Hospital da Polícia Militar General Edson Ramalho (HPMGER).

Para a coleta de dados, foram utilizados como critérios de inclusão os profissionais de Enfermagem que estavam em serviço no momento da aplicação do questionário e que concordaram em participar da pesquisa. Os profissionais que não estavam em serviço ou se encontravam afastados por algum motivo, durante o período do estudo, foram excluídos do procedimento.

Aos que aderiram à pesquisa disponibilizou-se o termo de consentimento livre e esclarecido (TCLE), sendo uma cópia entregue a cada colaborador, e explicou-se os objetivos da análise. Para a realização do estudo foram respeitados os preceitos éticos que regem as pesquisas com seres humanos, constantes na Resolução n. 466/2012 e CAAE número 47355315.3.0000.5183.

A partir da aceitação por parte dos profissionais, foi aplicado um questionário semiestruturado com perguntas de múltipla escolha, sendo que, em algumas delas, era possível assinalar mais de uma opção. O instrumento foi formulado contendo, na primeira parte, os dados relativos às características dos profissionais, como sexo, escolaridade, tempo de trabalho na instituição, tempo de trabalho exclusivamente no CME e existência de outro vínculo empregatício. A segunda parte, por sua vez, abordou dados como a utilização de EPI e sua frequência de uso, os tipos de EPI mais utilizados, o conhecimento sobre a quantidade e a qualidade dos equipamentos disponíveis pelo setor, os motivos que podem ocasionar a exposição dos profissionais aos riscos ocupacionais, os tipos de riscos ocupacionais a que estão expostos, a propensão à ocorrência de acidente de trabalho e de treinamento em serviço sobre prevenção de acidentes e a importância quanto aos treinamentos contínuos em serviço.
Os achados foram tabulados e apresentados em tabelas, transcritos com base nos dados colhidos. O processamento foi realizado a partir da utilização do software Windows Excel, por meio de estatística simples, e apresentado em números absolutos e percentuais. Dessa forma, houve melhora na compreensão dos achados pelos pesquisadores.

\section{RESULTADOS}

O estudo identificou 48 (96\%) entrevistados do sexo feminino e 2 (4\%) do masculino, constatou, ainda, que 13 (26\%) são enfermeiros, 18 (36\%) técnicos de Enfermagem e 19 (38\%) auxiliares de Enfermagem.

De acordo com o tempo de trabalho nas instituições, $16(32 \%)$ têm entre 1 e 5 anos, $5(10 \%)$ entre 6 e 10 anos e 29 (58\%) mais de 10 anos de experiência. Quanto ao tempo de trabalho especificamente nos CME, 17 (34\%) possuem entre 1 e 5 anos, 7 (14\%) entre 6 e 10 anos e $26(52 \%)$ mais de 10 anos. Entre os entrevistados, 32 (64\%) referiram possuir apenas um vínculo empregatício.

Em relação à frequência de utilização do EPI, 37 (74\%) mencionaram que o usam sempre que desenvolvem suas atividades, $11(22 \%)$ citaram que usam algumas vezes e 2 (4\%) não responderam a essa questão. Em relação aos EPI mais utilizados, as luvas, 48 (96\%), e as máscaras, 42 (84\%), foram as mais prevalentes, enquanto os capotes, 15 (30\%), os menos referidos pelos pesquisados (Tabela 1).

De acordo com as opiniões sobre os EPI disponíveis, 20 (40\%) entrevistados os consideraram sem qualidade, 13 (26\%) insuficientes, $11(22 \%)$ suficientes e $6(12 \%)$ de boa qualidade.

Tabela 1. Equipamentos de proteção individual utilizados por profissionais de Enfermagem em Centros de Material e Esterilização de dois hospitais públicos. João Pessoa, Paraíba. Brasil, 2016.

\section{Equipamentos de}

proteção individual

$\mathbf{N}$

Luvas

Máscara

Óculos

35

Avental

25

Gorro

35

Capote

15

Calçado fechado

38

n: número de profissionais entrevistados.
$\%$

96

84

70

50

70

30

76 
Os dados apontaram, ainda, que $34(68 \%)$ profissionais acreditam que os EPI disponíveis não são adequados para os procedimentos realizados nos CME. Trinta e um (62\%) entrevistados referiram a exposição aos riscos ocupacionais em decorrência de EPI inadequados e 4 ( $8 \%$ ) por desconhecimento do profissional (Tabela 2).

Em relação à vivência com algum tipo de exposição a risco ocupacional no CME, 37(74\%) entrevistados afirmaram que estão em risco e 13(26\%) não responderam.

Evidenciou-se que $38(76 \%)$ profissionais não sofreram acidente no trabalho, enquanto 12 (24\%) contrapuseram, relatando já ter sofrido algum tipo de acidente. Quanto à propensão aos tipos de acidente a que se consideram estar expostos, $29(58 \%)$ apontaram os acidentes com materiais perfurocortantes, $10(20 \%)$ as queimaduras, $4(8 \%)$ com soluções químicas e 7 (14\%) não referiram.

Observou-se também que 33 (66\%) afirmaram já ter realizado algum treinamento direcionado à prevenção de acidentes, 12 (24\%) informaram não ter realizado e 5 (10\%) não responderam a essa questão. No que se refere à necessidade de treinamentos sobre a prevenção de acidentes de trabalho, 49 (98\%) consideraram de suma importância o treinamento permanente com intuito de esclarecer a forma adequada de uso de EPI.

\section{DISCUSSÃO}

A pesquisa apontou prevalência do sexo feminino entre os entrevistados, constatação semelhante a que foi realizada com 37 profissionais de Enfermagem, atuantes em dois hospitais de referência, um público e um privado, ambos localizados na cidade de Caruaru, agreste pernambucano. Além disso, essa predominância é característica dessa profissão $0^{9,10}$.

Tabela 2. Motivação para exposição dos profissionais de Enfermagem aos riscos ocupacionais no Centro de Material e Esterilização. João Pessoa, Paraíba. Brasil, 2016.

\begin{tabular}{|l|c|}
\hline Causas de exposição & $\%$ \\
\hline EPI inadequados & 62 \\
\hline Considerar o uso desnecessário & 50 \\
\hline Falta de treinamento & 46 \\
\hline Falta de EPI & 32 \\
\hline Negligência do profissional & 28 \\
\hline Desconhecimento do uso & 8 \\
\hline
\end{tabular}

EPI: equipamento de proteção individual.
De acordo com o tempo de trabalho nas instituições percebeu-se a existência de profissionais com período longo de experiência no ambiente hospitalar, assim como no CME. Logo, a dimensão do conhecimento adquirido com os anos de experiência técnica específica no setor vem como uma qualidade para garantir a efetividade do serviço pela equipe de Enfermagem. A simetria com o estudo em pauta corrobora com uma pesquisa realizada em um hospital escola de Goiás, em que 28 (10,4\%) entrevistados indicaram a importância do conhecimento específico e da experiência do profissional para a obtenção de resultados satisfatórios e eficientes nas atividades laborais ${ }^{11}$.

Sabe-se que o uso de EPI como gorro, óculos, máscara, luvas grossas de borracha, avental impermeável e sapato fechado é uma forma de amenizar os riscos aos quais os trabalhadores estão expostos, como contaminação por respingos de sangue ou demais secreções corpóreas ou acidentes percutâneos, acometendo frequentemente os trabalhadores de Enfermagem, bem como aqueles que atuam nos $\mathrm{CME}^{8,9,12}$. No entanto, é lamentável perceber neste estudo que alguns profissionais não os utilizam frequentemente, expondo-se, assim, a riscos ocupacionais.

Pesquisadores revelaram em hospital da região CentroOeste que a autoconfiança leva ao descaso no uso dos EPI, sendo reforçada pela experiência de que seu uso interfere nas habilidades do profissional e dificulta a execução do procedimento. Assim, o profissional opta por não usá-los, subestimando a sua função de proteção ${ }^{8}$. Esse resultado concorre com o que acontece nos CME avaliados, nos quais 14 profissionais reconheceram ser negligentes quanto ao não uso dos EPI.

Isso nos faz refletir sobre a indiferença com as medidas de biossegurança, conceitos simples, mas que protegem, eliminam ou minimizam os riscos existentes, buscando como finalidade a saúde do homem e dos animais e a preservação do meio ambiente ${ }^{13}$. Dessa forma, é indispensável o uso do EPI, os quais deverão ser ofertados de forma gratuita, conforme a Norma Regulamentadora do Ministério do Trabalho (NR) 6, para garantir a segurança dos que estão expostos aos riscos de perfuração ou corte, prevenindo acidentes de trabalho ou doenças ocupacionais 4 .

Destaca-se o resultado de uma pesquisa realizada em um hospital de Londrina, Paraná, na qual foi constatado que os trabalhadores fazem uso dos EPI em decorrência de normas exigidas pelas instituições em que trabalham. Logo, pode-se cogitar que, se pudessem escolher, esses profissionais não os usariam. Ainda, os mesmos trabalhadores (100\%) não têm 
poder de escolher o tipo/modelo do EPI que melhor se adapta às suas atividades, pois já existe a preconização do EPI a ser utilizado nos locais em que trabalham ${ }^{14}$. Dessa forma, tal descoberta assemelha-se aos dados desta pesquisa, nos quais mais da metade dos entrevistados apontou a existência de EPI inadequados aos procedimentos. Tal fato alerta para a situação de risco a que os profissionais podem estar expostos, pois, uma vez não adequados às atividades, possivelmente não oferecerão a segurança necessária.

Assim, os recursos materiais devem oferecer ao trabalhador segurança durante o desenvolvimento de suas atividades, considerando os aspectos de qualidade e quantidade suficientes, como forma de não desqualificar o trabalho realizado para que outros profissionais possam ser prejudicados, trazendo, assim, algum risco ocupacional ${ }^{5}$.

A exposição ocupacional acidental entre os profissionais de saúde é constante em decorrência do número exagerado de manipulação e representa prejuízos à saúde dos trabalhadores e às próprias instituições ${ }^{15}$. Dessa forma, as causas dos acidentes de trabalho apontaram similaridades à realidade encontrada em outro estudo que aborda essa temática, realizado em dois hospitais do Nordeste, no qual $83,3 \%$ dos acidentes ocorreram devido a materiais perfurocortantes e $57,1 \%$ por queimaduras em autoclave ${ }^{9}$.

No tocante à realização de treinamentos e considerando que a maioria dos profissionais afirmou ter realizado capacitação sobre a prevenção de acidentes, reforça-se a ideia de que essa é uma das estratégias mais usadas para enfrentar os desafios de desenvolvimento dos serviços de saúde. A educação permanente vem sendo utilizada como meio para o desenvolvimento de competências e habilidades dos trabalhadores. Tendo-se em vista a abrangência e a importância do CME no contexto hospitalar, enaltece-se a necessidade de práticas educativas contínuas aos que atuam nessa área ${ }^{14}$.

Divergindo desta pesquisa, um estudo realizado com 38 profissionais de Enfermagem de seis municípios do Estado do Ceará, que objetivou traçar a necessidade de capacitação, revelou carências significativas em relação às atualizações e aos treinamentos, problemática passível de reversão se houver treinamentos adequados ${ }^{16}$. A disponibilidade dos profissionais de acesso à informação sobre os assuntos pertinentes ao processo de trabalho possibilita a evolução técnica necessária aos serviços de saúde, para que esses sujeitos sejam capazes de desempenhar suas funções de forma competente, eficaz e sem riscos para cada profissional.

Ainda nesse contexto, essa necessidade de formação, qualificação e atualização dos profissionais atuantes em CME pode proporcionar um trabalho de qualidade, desde que investigados os aspectos da prática profissional, sinalizando problemas identificados, muitas vezes ligados aos "vícios" da rotina da profissão. Tal situação remete ao tempo de atuação no setor com profissionais, como mais da metade dos entrevistados, que têm acima de 10 anos de trabalho.

Uma pesquisa realizada em três hospitais públicos do Rio de Janeiro, quanto à importância da participação do trabalhador em programas de educação continuada, revelou que, devido às peculiaridades das atividades desenvolvidas no CME, há dificuldades de se manter um grupo estável e em sincronia com o serviço, sendo preciso reverter ou prevenir essa situação, desenvolvendo-se programas contínuos de treinamento e aperfeiçoamento dos trabalhadores ${ }^{17}$. Determinado resultado diverge com o desta pesquisa, visto que a maioria dos profissionais atuantes nos CME dos dois hospitais trabalha no setor há um tempo relevante, o que demonstra um grupo permanente nesse serviço.

\section{CONCLUSÃO}

Com a realização deste estudo, foi possível identificar o perfil profissional no setor de CME dos hospitais pesquisados, sendo o sexo feminino o mais predominante, o técnico de Enfermagem a categoria profissional mais frequente e a maioria dos entrevistados com mais de 10 anos de experiência e apenas um vínculo empregatício.

Comprovou-se que a grande maioria faz uso frequente de EPI, em especial as luvas, porém, consideram que os materiais disponibilizados são de pouca qualidade e, por vezes, não se adaptam às atividades desenvolvidas no setor.

Observou-se que muitos profissionais estiveram expostos a algum tipo de risco ocupacional e reconheceram que nem sempre fazem uso dos equipamentos de forma adequada, por considerarem desnecessário ou por simples negligência pessoal.

Treinamentos realizados sobre a temática são identificados, porém reconhecem a necessidade de educação permanente sobre o assunto durante o turno de trabalho como forma de aprimorar o conhecimento.

Diante do exposto, os resultados apontam para a necessidade de realização de uma educação continuada e permanente como estratégia das medidas preventivas no intuito de sensibilizar os profissionais quantos aos cuidados necessários e à importância do uso de EPI, visando evitar os acidentes em CME. 


\section{REFERÊNCIAS}

1. Soares LG, Sarquis LMM, Kirchhof ALC, Felli VEA. Multicausalidade nos acidentes de trabalho da Enfermagem com material biológico. Rev Bras Enferm [Internet]. 2013 [acesso em 11 fev. 2017];66(6):854-9. Disponível em: http://www.scielo.br/scielo. php?script=sci_arttext\&pid=S0034-71672013000600007\&lng=en

2. Bezerra VS, Santana RS, Brito BAM, Ferreira JLS, Gama MEA, Maslinkiewicz A. Riscos biológicos enfrentados pelos enfermeiros na Unidade de Terapia Intensiva. Rev Pre Infec Saúde [Internet]. 2015 [acesso em 15 dez. 2015];1(3):18-26. Disponível em: http://revistas. ufpi.br/index.php/nupcis/article/view/4231/pdf

3. Marziale MHP, Rocha FLR, Robazzi MLDCC, Cenzi CM, Santos HEC, Trovó MEMP. Influência organizacional na ocorrência de acidentes de trabalho com exposição a material biológico. Rev Latino-Am Enferm [Internet]. 2013 [acesso em 10 maio 2016];21:1-8. Disponível em: https://scholar. google.com.br/scholar?q=Influ\%C3\%AAncia+organizacional+na+ocorr $\%$ C3\%AAncia+de+acidentes+de+trabalho+com+exposi $\%$ C3\%A7\%C3\% $\mathrm{A} 30+a+$ material+biol\%C3\%B3gico\&btnG $=\& h l=p t-B R \& a s \_s d t=0 \% 2 C 5$

4. DiehL D, Rosa K, Rosa SS, Krug SBF. Notificações de acidentes de trabalho com material biológico: um estudo no município de Santa Cruz do Sul/RS. Rev Epidemiol Control Infect [Internet]. 2012 [acesso em 15 dez. 2015];2(3):85-8. Disponivel em: https://online.unisc.br/ seer/index.php/epidemiologia/article/view/2707/2189

5. Magagnini AM, Rocha AS, Ayres JA. O significado do acidente de trabalho com material biológico para os profissionais de enfermagem. Rev Gaúcha Enferm [Internet]. 2011 [acesso em 30 maio 2016];32(2):302-8. Disponível em: http://dx.doi.org/10.1590/S1983-14472011000200013

6. Araruna AB, Posso MBS. Centro de material de esterilização: parâmetros espaciais e riscos físicos. Rev SOBECC [Internet]. 2014 [acesso em 12 set. 2016];19(3):142-7. Disponível em: http://www.sobecc.org.br/ arquivos/artigos/2015/pdfs/site_sobecc_v19n3/05_sobecc.pdf

7. Pereira FMV, Toffano SEM, Silva AM, Canini RMS, Gir E. Rev Esc Enferm USP [Internet]. 2013 [acesso em 12 set. 2016];47(3):681-7. Disponível em: http://www.revistas.usp.br/reeusp/article/view/78012/82001

8. Neves HCC, Souza ACS, Medeiros M, Munari DB, Ribeiro LCM, Tipple AFV. Segurança dos trabalhadores de enfermagem e fatores determinantes para adesão aos equipamentos de proteção individual. Rev Latino-Am Enferm [Internet]. 2011 [acesso em 12 set. 2016];19(2). Disponivel em: http://www.scielo.br/pdf/rlae/v19n2/pt_18

9. Aquino M, Barros LP, Brito AS, Ferreira EB, Medeiros SEG, Santos ER. Centro de material e esterilização: acidentes de trabalho e riscos ocupacionais. Rev SOBECC [Internet]. 2014 [acesso em 12 set. 2016];19(3):148-54. Disponível em: http://www.sobecc.org.br/ arquivos/artigos/2015/pdfs/site_sobecc_v19n3/06_sobecc.pdf
10. Tanabe LP, Kobayashi RM. Perfil, competências e fluência digital dos enfermeiros do Programa de Aprimoramento Profissional. Rev Esc Enferm USP [Internet]. 2013 [acesso em 14 set. 2016];47(4):943-9. Disponivel em: http://www.scielo.br/scielo. php?script=sci_arttext\&pid=S0080-62342013000400943\&lng=pt

11. Martins VMF, Munari DB, Tipple ACFV, Bezerra ALQ, Leite JL, Ribeiro LCM. Forças impulsoras e restritivas para trabalho em equipe em um Centro de Material e Esterilização de hospital escola. Rev Esc Enferm USP [Internet]. 2011 [acesso em 16 set. 2016];45(5):1183-90. Disponível em: http://www.scielo.br/scielo. php?script=sci_arttext\&pid=S0080-62342011000500022\&lng=en

12. Simonelli AP, Jackson Filho JM, Vilela RAG, Almeida IM. Influência da segurança comportamental nas práticas e modelos de prevenção de acidentes do trabalho: revisão sistemática da literatura. Saúde Socied [Internet]. 2016 [acesso em 14 set. 2016];25(2):463-78. Disponível em: http://www.scielo.br/scielo. php?script=sci_arttext\&pid=S0104-12902016000200463\&lng=en

13. Brasil. Ministério da Saúde. Política Nacional de Educação Permanente em Saúde. Departamento de Gestão da Educação em Saúde. Brasília: Ministério da Saúde; 2009. V. 9. Disponível em: http://portal. anvisa.gov.br/documents/33856/396770/Pol\%C3\%ADtica+Naciona l+de+Educa\%C3\%A7\%C3\%A30+Permanente+em+Sa\%C3\%BAde/ c92db117-e170-45e7-9984-8a7cdb111 faa

14. Ribeiro RP, Vianna LAC. Uso de equipamentos de proteção individual entre trabalhadores das centrais de material e esterilização. Ciênc Cuid Saúde [Internet]. 2012 [acesso em 1.º set. 2016]. Disponível em: http://www.periodicos.uem.br/ojs/index.php/CiencCuidSaude/ article/view/17076

15. Camilo ENR, Arantes TEFH, Lemos S. Epidemiological analysis of accidents with biological material in an eye hospital. Rev Bras Oftalmol [Internet]. 2015;74(5):284-7. Disponível em: http://www.scielo.br/pdf/rbof/v74n5/en_0034-7280rbof-74-05-0284.pdf

16.Ximenes Neto FRG, Costa MCF, Rocha J, Cunha ICKO. Auxiliares e técnicos de enfermagem na saúde da família: perfil sociodemográfico e necessidades de qualificação. Trab Educ Saúde [Internet]. 2008 [acesso em 16 set. 2016];6(1):51-64. Disponível em: http://www.scielo.br/scielo. php?script=sci_arttext\&pid=S1981-77462008000100004

17. Pezzi MCS, Leite JL. Investigação em Central de Material e Esterilização utilizando a Teoria Fundamentada em Dados. Rev Bras Enferm [Internet]. 2010 [acesso em 15 set. 2016];63(3):391-6. Disponível em: http://www.scielo.br/scielo. php?script=sci_arttext\&pid=S0034-71672010000300007\&lng=en 